\title{
Exotics searches in top, top-like and diboson final states with the ATLAS detector
}

\section{Thorsten KUHL*}

DESY, Platanenalle 6, 15738 Zeuthen

E-mail: Thorsten.Kuhlecern.ch

We present analyses for top quark and top quark like final states using an integrated luminosity of 33 to $200 \mathrm{pb}^{-1}$ of data recorded with the ATLAS experiment. The analyses include searches for resonances decaying into $t \bar{t}$, top-pair final states with anomalous missing energy, and a search for fourth generation quarks decaying top-like. Cross section limits are shown for the production of an heavy $Z^{\prime}$ decaying into $t \bar{t}$. In Randall-Sundrum models, Kaluza-Klein gluons with masses below $650 \mathrm{GeV}$ are excluded at $95 \%$ C.L. In the context of quantum black holes, enhanced $t+\mathrm{X}$ production with a mass threshold below $2.35 \mathrm{TeV}$ is excluded. Exclusion limits of $275 \mathrm{GeV}$ and $300 \mathrm{GeV}$ are set for a heavy top decaying into a neutral particle with mass less than $50 \mathrm{GeV}$ and less than $10 \mathrm{GeV}$, respectively. We exclude at $95 \%$ confidence level a heavy decaying quark with mass less than $270 \mathrm{GeV}$.

The 2011 Europhysics Conference on High Energy Physics-HEP 2011,

July 21-27, 2011

Grenoble, Rhône-Alpes France

\footnotetext{
* Speaker.
} 


\section{Introduction}

The Standard Model of particle physics is believed to be an effective theory valid up to energies close to $1 \mathrm{TeV}$. However, no new physics beyond the Standard Model have been observed yet, and it is critical to explore a wide range of possible signatures. A promising venue lies in final states that involve the heaviest of the particles presumed to be elementary, the top quark. Many models predict heavy resonances which couples to top quarks strongly like Kaluza Klein gluons [4, 5, 6, 7] or weakly like a $Z^{\prime}$ [3]. Also there are decays of heavy new particles into top quarks [13] or top quark like decaying new particles [15].

The LHC had a very successful data taking in 2011 and a delivered luminosity of about $1.3 \mathrm{fb}^{-1}$ till the EPS conference and more than $4.5 \mathrm{fb}^{-1}$ since the end of September. In this proceedings results based on a data set between 33 and $200 \mathrm{pb}^{-1}$ are shown. The events in the data set were collected with the ATLAS detector [1] at the LHC in 2010 and 2011 mostly using single lepton triggers [2] with thresholds at 20 and $18 \mathrm{GeV}$ for the electron and muon channels, respectively.

\section{Results}

Most of these searches are based on the semileptonic signature, where one of the two top quarks is decaying into three jets and the other one in a jet plus an electron or muon in association with a neutrino from the $\mathrm{W}$ boson decay. Therefore the selections of events for the different search channels are similar. First a high-energy isolated lepton with a momentum threshold of $20-25 \mathrm{GeV}$ is selected. The event should have missing transverse energy and the transverse lepton-neutrino mass should be in agreement with the expectation from a W boson decay. In the last step 4 or more jets of $20-25 \mathrm{GeV}$ are selected.

For resonant $t \bar{t}$ production in the lepton plus jets final states results with an integrated luminosity of $200 \mathrm{pb}^{-1}$ are shown [9]. Figure 1 left shows the invariant mass distribution for the background (yellow) and the data (dots). Additionally two example signal distributions, one for an $Z^{\prime}$ of $m_{t t}=$ $500 \mathrm{GeV}$, a narrow weakly couple resonance and one for an Kaluza Klein Gluon of $m_{t t}=1000 \mathrm{GeV}$, a wide strongly couple resonance are shown. For the reconstruction of the di-top mass $m_{t t}$ all jets above $p_{t}>20 \mathrm{GeV}$ are used. Jets which are well isolated from all other selected objects are rejected and the mass is reconstructed from the remaining 4 jets with the highest momentum or 3 jets, if only 3 survive this procedure. This algorithm primary helps to remove jets from initial state radiation, which are mostly isolated jets and more in forward direction. Limits are set on the production crosssection times branching ratio to $t \bar{t}$ for narrow and wide resonances. For narrow $Z^{\prime}$ models, the limit is shown in Figure 1 right. The observed 95\% C.L. limits range from approximately $38 \mathrm{pb}$ to $3.2 \mathrm{pb}$ for masses between $m_{Z^{\prime}}=500 \mathrm{GeV}$ to $m_{Z^{\prime}}=1300 \mathrm{GeV}$. In Randall-Sundrum models, Kaluza-Klein gluons with masses below $650 \mathrm{GeV}$ are excluded at $95 \%$ C.L.. Previously [8] a limit on enhanced top quark production at high $t+X$ mass ( $X$ any additional object produce in association with the top quark), using the production of quantum black holes [10] to model the signal was shown. In that context, enhanced $t+X$ production with a mass threshold below $2.35 \mathrm{TeV}$ is excluded.

With increasing invariant mass of the resonant structure $m_{t t}$ the top-quarks get highly boosted and the decay products of the top quarks are closer together. Finally the jets and leptons of the decay 

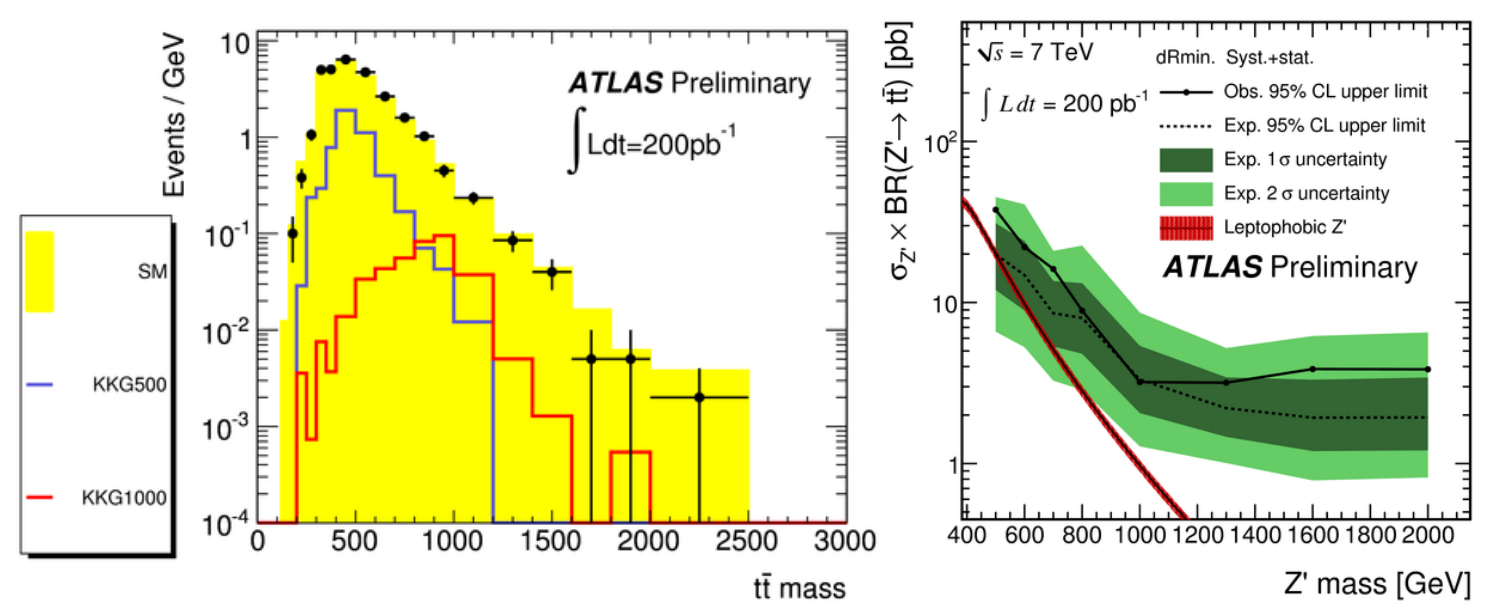

Figure 1: Distribution of the reconstructed di-top mass $m_{t t}$ (left). Expected and observed cross section limit for a $\mathrm{Z}$ in the $t \bar{t}$ resonance search.

products merge into a single large jet. Initial studies for big jet structures were presented [11]. One of the biggest problem is the additional energy deposited in the big jet cone by pile up interaction in these events. The studies show that in average this additional energy has a well defined behavior dependent on the number of pile up events and can be calibrated.

A signature similar to that of the resonant di-top production is the production of two top quarks in association with anomalous missing transverse energy [12]. Such a phenomenon can arise from a number of Standard Model extensions. One possible candidate is the production of a heavy top quark decaying into a top quark plus a heavy stable neutral particle [13]. Different to the $t \bar{t}$ resonance analysis additional large missing transverse energy is expected. In the first LHC data with a luminosity of $35 \mathrm{pb}^{-1}$ an analysis focusing on a search for a pair-produced top partner decaying to a top quark and a long-lived neutral particle which escapes undetected was performed. Figure 2 (left) shows the distribution of the reconstructed mass of the top plus missing transverse energy final state. No excess in the data is observed. Limits are set excluding a mass of $275 \mathrm{GeV}$ for a neutral particle mass less than $50 \mathrm{GeV}$ and a mass of $300 \mathrm{GeV}$ for a neutral particle mass less than $10 \mathrm{GeV}$.

The last analysis which is presented is a search for pair production of a fourth generation quark [14]. A decay similar to the top quark was investigated where the fourth generation quark [15] decays into a light quark $\mathrm{q}(\mathrm{q}=\mathrm{u}, \mathrm{d}, \mathrm{c}, \mathrm{s}, \mathrm{b})$ plus a $\mathrm{W}$ boson. This analysis was performed in the di-lepton channel where both $\mathrm{W}$ bosons decay leptonically. Therefore a mass reconstruction assuming that the neutrino has the same flight direction as the leptons was applied. Figure 2 shows the expected and observed limit for this process. With $37 \mathrm{pb}^{-1}$ of integrated luminosity we exclude at $95 \%$ confidence level a heavy quark with mass less than $270 \mathrm{GeV}$.

\section{Summary}

We presented analyses for top quark and top quark like final states using an integrated luminosity of 33 to $200 \mathrm{pb}^{-1}$ of data recorded with the ATLAS experiment. The analyzes include searches 

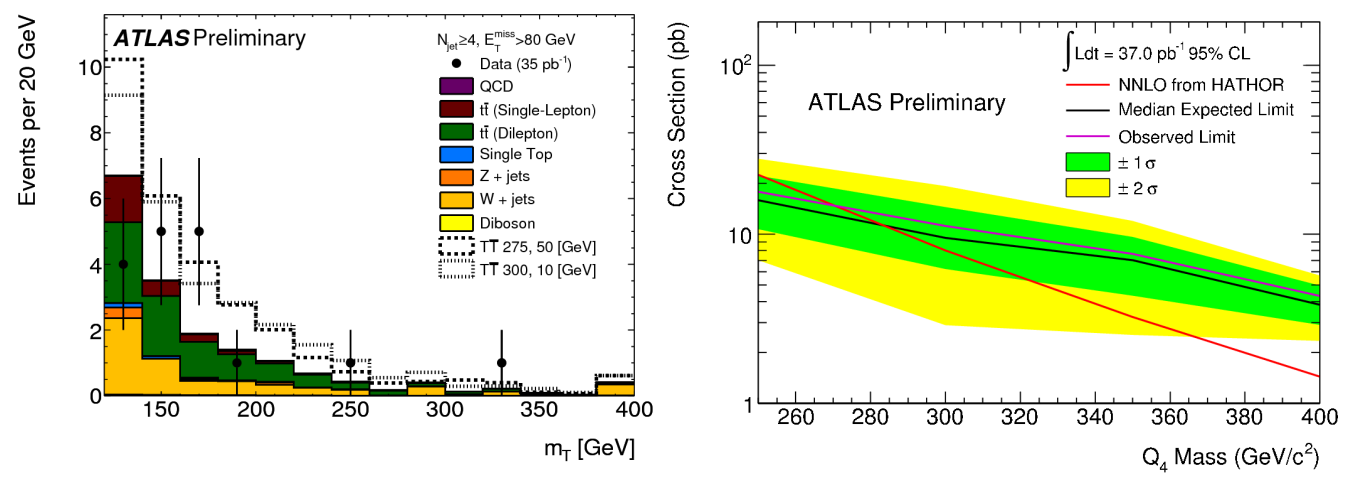

Figure 2: Distribution of the the Di-Top transverse mass (left) in the search for $t \bar{t}$ plus missing energy and the limit for the fourth generation seach (right).

for resonances decaying into $t \bar{t}$, top quark pair final states with anomalous missing energy, and a search for fourth generation quarks. Cross section limits were shown for the models.

\section{References}

[1] G. Aad et al., JINST 3 (2008) S08003.

[2] G. Aad et al., JHEP 1012 (2010) 060.

[3] R. M. Harris, C. T. Hill and S. J. Parke, arXiv:hep-ph/9911288.

[4] B. Lillie, L. Randall, L. -T. Wang, JHEP 0709 (2007) 074.

[5] A. Djouadi, G. Moreau and R. K. Singh, Nucl. Phys. B 797 (2008) 1.

[6] B. C. Allanach, F. Mahmoudi, J. P. Skittrall, K. Sridhar, JHEP 1003 (2010) 014.

[7] ATLAS collaboration, "Prospects for top anti-top resonance searches using early ATLAS data.", ATL-PHYS-PUB-2010-008, CERN, Geneva, Jul, 2010.

[8] ATLAS collaboration, "A Search for $t \bar{t}$ Resonances in the Lepton Plus Jets Channel in $200 \mathrm{pb}^{-1}$ of $p p$ Collisions at $\sqrt{s}=7 \mathrm{TeV} "$, ATLAS-CONF-2011-087, CERN, Geneva, June, 2011.

[9] ATLAS collaboration, "A Search for New High-Mass Phenomena Producing Top Quarks with the ATLAS Experiment", CERN, Geneva, May, 2011.

[10] P. Meade, L. Randall, JHEP 0805 (2008) 003.

[11] ATLAS collaboration, "Measurement of Jet Mass and Substructure for Inclusive Jets in ấŽs $=7 \mathrm{TeV}$ pp Collisions with the ATLAS Experiment", ATLAS-CONF-2011-073, CERN, Geneva, May, 2011.

[12] ATLAS collaboration, "Search for Anomalous Missing ET in tt Events", ATLAS-CONF-2011-036, CERN, Geneva, March, 2011.

[13] J. Alwall, J. L. Feng, J. Kumar, S. Su, Phys. Rev. D81 (2010) 114027.

[14] ATLAS collaboration, "Search for Fourth Generation Quarks Decaying to $W q W q \rightarrow l l v v q q$ in $p p$ collisions at $\sqrt{s}=7 \mathrm{TeV}$ with the ATLAS Detector", ATLAS-CONF-2011-022, CERN, Geneva, March, 2011.

[15] J. A. Aguilar-Saavedra, JHEP 0911, 030 (2009) 